\title{
A Case of Adult Filipino Male Presenting with Co-localized Psoriasis and Segmental Vitiligo
}

\author{
Shahara Abalos-Babaran ${ }^{1}$ and Ma. Lorna F. Frez ${ }^{2}$ \\ ${ }^{1}$ Section of Dermatology, Department of Medicine, Philippine General Hospital, University of the Philippines Manila \\ ${ }^{2}$ Section of Dermatology, Department of Medicine, College of Medicine and Philippine General Hospital, University of the Philippines Manila
}

\begin{abstract}
A 29-year-old male presented with a four-year history of recurrent generalized pruritic erythematous scaly annular plaques followed by a solitary depigmented patch on the right hemiabdomen extending to the back with abrupt midline demarcation. Skin biopsies confirmed the rare case of co-existing and colocalized psoriasis and segmental vitiligo. Treatment with topical steroids, tacrolimus, and narrowband ultraviolet B phototherapy resulted to the improvement of the psoriatic lesions but no change in the depigmented patch.
\end{abstract}

Key Words: psoriasis, segmental vitiligo, vitiligo

\section{INTRODUCTION}

Vitiligo and psoriasis are both common conditions in the general population. Hence, the concurrence of both is likely based on their incidences ${ }^{1}$ and similar predilection sites. $^{2}$ But strict anatomical colocalization of lesions is very rare. ${ }^{3}$ This is the first report worldwide of coexistence of psoriasis and segmental vitiligo with anatomical colocalization.

\section{CLINICAL CASE}

This is a case of a 29-year-old male, single, a computer technician from Pasay City with a four-year history of recurrent erythematous pruritic scaly plaques starting on the scalp with spread to the face, trunk, and extremities. Spontaneous resolution of most of these was observed. Two years later, he noted asymptomatic depigmented macules coalescing into a single patch over the right hemiabdomen extending to the back. Pruritic erythematous annular plaques appeared over the depigmented patch one month prior to consult. There was no arthralgia. Family history was positive for psoriasis and vitiligo.

On physical examination, there were generalized, well-

E-poster presented at the $41^{\text {st }}$ Annual Convention of the Philippine Dermatological Society, November 7-9, 2018, EDSA Shangri-La, Manila, Philippines.

Corresponding author: Shahara Abalos-Babaran, MD Section of Dermatology

Department of Medicine Philippine General Hospital

University of the Philippines Manila

Taft Avenue, Manila 1000, Philippines

Telephone: +632 5548400 local 5105/5106

Email: shahara_731@yahoo.com demarcated erythematous annular and arcuate plaques with coarse white scales. A solitary irregularly-shaped depigmented patch on the right abdomen extending to the back with abrupt midline demarcation was noted. Some erythematous, irregularly-shaped plaques and islands of normo-pigmented skin were observed within this depigmented patch. The palms, soles, and genitals were spared. Multiple pits were seen on several fingernails. Lastly, there were multiple lentigines noted on the trunk and feet. Wood's lamp examination of the depigmented patch showed 

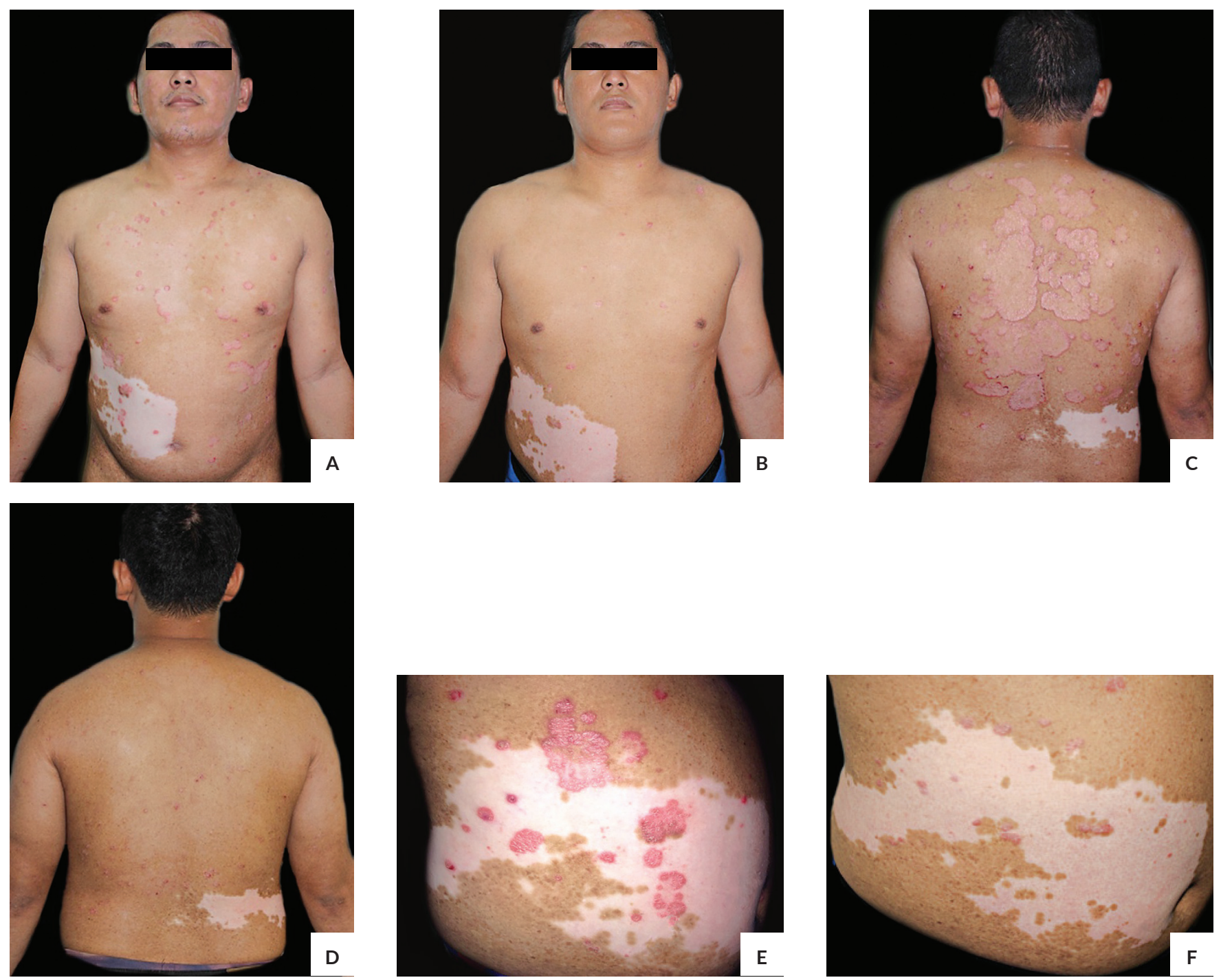

Figure 1. Multiple erythematous well-defined annular and arcuate plaques with coarse white scales on the face, trunk and extremities with a solitary depigmented patch on the right hemiabdomen extending to the back as seen in $\mathbf{A}$ and $\mathbf{C}$. A closer view of the solitary depigmented patch with islands of normopigmented skin within and overlying erythematous well-defined plaques with silvery scales (E). After two months of narrowband UVB combined with topical steroid and tacrolimus (B, D, F) show marked the improvement of psoriatic lesions however with no change in the vitiliginous patch.

a bright white and sharply delineated lesion. These findings are shown in Figures 1 (A,C,E) and 2 (A,C,D).

Punch biopsies of three skin lesions were done. Microscopic examination of sections taken from the erythematous plaques located outside and within the depigmented patch on the abdomen showed confluent parakeratosis, hypogranulosis, regular psoriasiform hyperplasia, suprapapillary plate thinning, dilated blood vessels within the dermal papille and sparse superficial perivascular lymphohistiocytic infiltrates consistent with psoriasis seen in Figure 2(E). The biopsy from the depigmented patch showed sparse melanocytes in the basal layer shown in Figure 2(F). The sections from the depigmented patch and erythematous plaque within this patch were both negative for Melanoma Antigen Recognized by $\mathrm{T}$ cells 1 (Mart- 1 ) stain confirming the diagnosis of vitiligo.

Screening for concomitant autoimmune conditions using anti-nuclear antibody and rheumatoid factor tests were both negative. Thyroid function, blood chemistry, complete blood count and urinalysis were within normal limits. Lipid profile showed slight hypertriglyceridemia $(179.09 \mathrm{mg} / \mathrm{dL})$. The rapid plasma reagin test was non-reactive.

The patient was advised to apply fluticasone acetonide $0.025 \%$ cream and clobetasol propionate $0.05 \%$ cream twice a day for two weeks on the erythematous plaques on the face and trunk/extremities, respectively. The vitiliginous skin was treated with tacrolimus $0.01 \%$ ointment twice a 

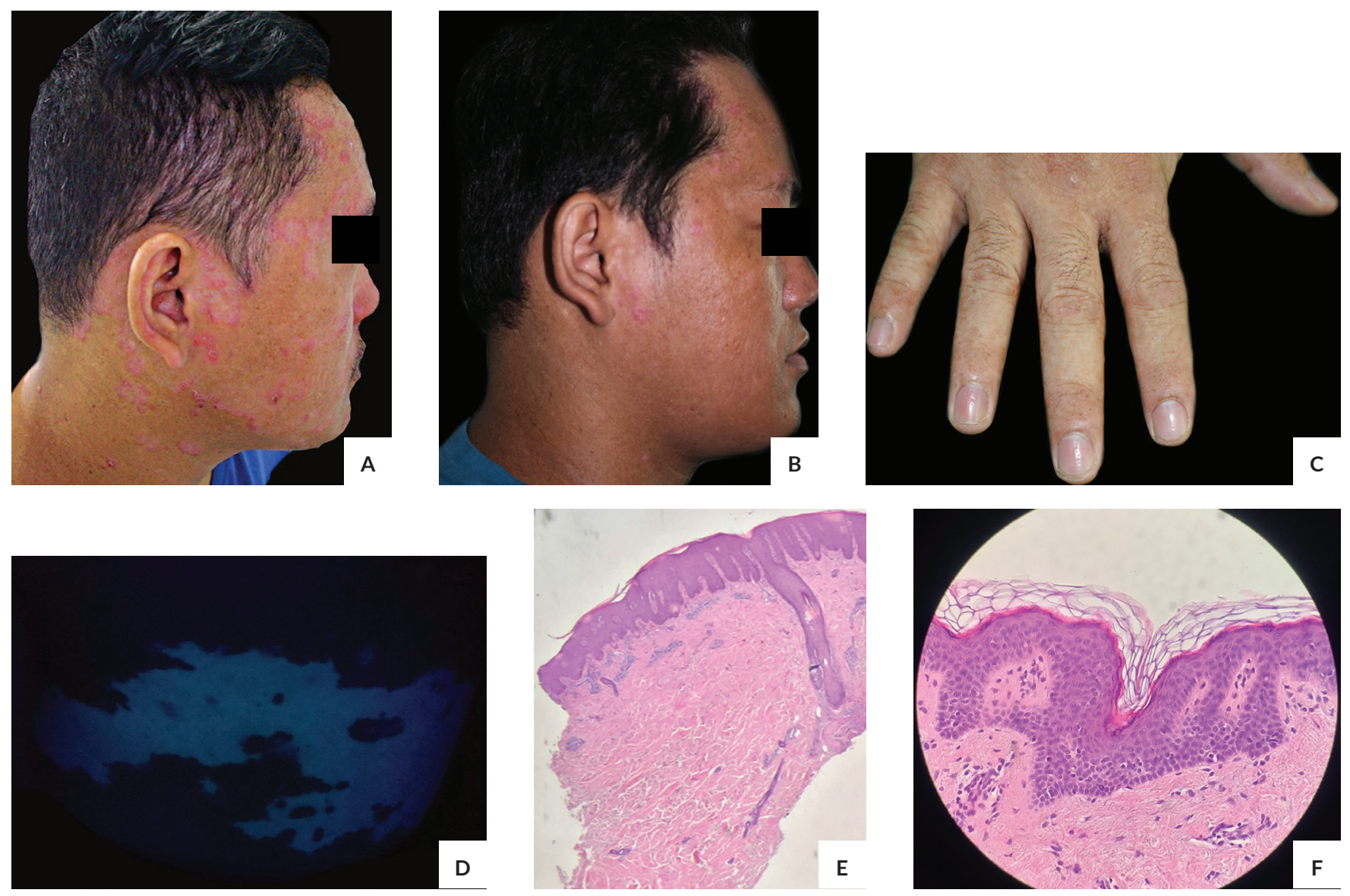

Figure 2. (A) shows a closer view of the erythematous annular scaly plaques on the face with the involvement of the scalp as seen in lateral view. After two months of narrowband UVB combined with a topical steroid (B) showing a decrease in the number of psoriatic lesions. Multiple irregulars and deep pits on several fingernail plates as seen in (C). Wood's lamp examination (D) showing bright white and sharply delineated patch. Scanning view of the biopsy taken from the erythematous plaque $(\mathrm{H \& E}, \mathrm{x} 10)$ on the abdomen outside the depigmented patch shows (E) confluent parakeratosis, hypogranulosis, regular psoriasiform hyperplasia, and suprapapillary plate thinning. Findings consistent with psoriasis. Biopsy ( $H \& E, x 40)$ of the solitary depigmented patch showing very sparse melanocytes on the basal layer of the epidermis (F) consistent with vitiligo.

day in alternate with clobetasol propionate $0.05 \%$ ointment. Application of mineral oil on the scalp at night and liberal bland emollients use on the skin were also emphasized.

Minimal erythema dose (MED) was determined on the vitiliginous skin $\left(200 \mathrm{~mJ} / \mathrm{cm}^{2}\right)$. Phototherapy using narrowband ultraviolet B at $70 \%$ of MED $\left(140 \mathrm{~mJ} / \mathrm{cm}^{2}\right)$ with increments of $10 \%$ as tolerated thrice a week was done. After two months of phototherapy with a cumulative dose of $7845 \mathrm{~mJ} / \mathrm{cm}^{2}$, excellent results were achieved for the psoriatic lesions. There was the resolution of most of the erythematous plaques on the scalp, face, extremities and trunk including the ones on the vitiliginous skin as seen in Figures 1(B), 1(D), 1(F) and 2(B). However, there was no repigmentation with stable extent of the vitiliginous patch (Figure $1 \mathrm{~F}$ ). There were episodes of erythema and burning pain on the vitiliginous patch managed with topical steroid and adequate UVB dose adjustment.

\section{DISCUSSION}

\section{Pathophysiology of psoriasis}

Psoriasis is regarded as an autoimmune disease but no true autoantigen has been definitely identified. Stimulus such as trauma, infection, drugs, stress and smoking in a genetically predisposed individuals incites keratinocyte injury releasing cytokines i.e. interleukin-1 $\beta$ (IL-1 $\beta$ ) and tumor necrosis factor (TNF) which activate dendritic cells. ${ }^{4}$ These cells migrate to the skin-draining lymph nodes to present an unknown antigen (either of self or of microbial origin) to naive $\mathrm{T}$ cells promoting their differentiation. Subsequently, T-helper 17 secretes IL-17 which stimulate keratinocyte hyperproliferation observed clinically as erythematous plaques with thick white to silvery scales. 


\section{Pathophysiology of vitiligo}

Vitiligo is subtyped based on their different clinical patterns: non-segmental and segmental type. Non-segmental vitiligo is frequently associated with a number of autoimmune diseases. This association with immunologic disorders led to the hypothesis that this type is an autoimmune process by itself and to the identification of antimelanocyte antibody ${ }^{5}$ causing autoantibody-dependent and direct cellular toxicity against melanocytes. ${ }^{6}$ Meanwhile, segmental vitiligo is caused by somatic mosaicism from de novo mutations in genes that are vital for melanocyte development.

\section{Pathophysiology of co-existing psoriasis and vitiligo}

The proposed pathogenic links of the two diseases are based on the account that the non-segmental type was the more reported variant coexisting with psoriasis.

The key cytokine seen in psoriatic lesions, TNF- $\alpha$ has also been found in huge amounts in lesional and perilesional skin of patients afflicted by vitiligo. ${ }^{7} \mathrm{TNF}-\alpha$ is supposed to cause depigmentation by indirect stimulation of B-cell activation increasing autoantibody against melanocytes, directly inducing apoptosis of melanocytes and impediment of melanogesis by its inhibitory effect on tyrosinase. ${ }^{8}$ Moreover, interleukin 17A, also seen in psoriasis lesion was also found to be elevated in serum and lesional skin among vitiligo patients. ${ }^{9}$ The increased levels of these cytokines are associated with T-cell-driven immune diseases which may explain the development of psoriatic lesions within vitiliginous patches in a genetically susceptible individual.

Koebner phenomenon is characterized by the appearance of lesions on healthy skin subjected to local trauma. In psoriasis, the trauma-induced lesions may be secondary to release of cytokines from damaged keratinocytes. In vitiligo, this may be initiated by the separation and transpidermal elimination of melanocytes caused by local injury ${ }^{10}$ and release of melanocyte antigenic components leading to the production of autoantibodies. ${ }^{11}$ This process may explain the coexistence of both disease, but it is most reasonable when vitiligo and psoriasis occurs within a parallel time interval ${ }^{12}$ and in locations frequently predisposed to trauma such as chronic minor friction. ${ }^{11}$

In 1998, Dhar et al. supposed that there are similarities between anti-stratum corneum antibodies and antimelanocyte antibodies. ${ }^{10}$ However, there is still no further studies providing concrete evidence to support this.

\section{Pathophysiology of Psoriasis and Segmental Vitiligo}

In 2005, de Castro described the prevalence rate of psoriasis among vitiligo patients identifying only one case of segmental vitiligo with associated psoriasis. Psoriasis preceded the segmental vitiligo in this patient, and no cutaneous colocalization was noted. ${ }^{13}$ The case discussed here is the second case report of concurrent psoriasis and segmental vitiligo. Moreover, to the best of our knowledge, our case is the first reported coexistence of chronic plaque type psoriasis and segmental vitiligo with anatomical colocalization.

At present, the possible link between psoriasis and segmental vitiligo has not yet been defined due to the scarcity of cases. Segmental vitiligo occurs mostly on the ventral side representing the migration pattern of the melanoblasts during embryogenesis as they come from the neural crest. ${ }^{14}$ A number of melanoblasts move dorsolaterally and multiply as they migrate through the dermis until they get to the ventral midline. In effect, this high mitotic activity puts the melanocytes at the ventral side of the body at substantial risk for developing somatic mosaicism until they reach the epidermis or hair follicle. ${ }^{15}$

The recent combination theory puts together somatic mosaicism and autoimmunity as the etiology for segmental vitiligo. The foremost step involves the secretion of inflammatory factors, neuropeptides, and catecholamines as inciting factors from stress or trauma. ${ }^{14}$ In our patient, the psoriatic flare observed mostly on the trunk (caused by koebnerization from frequent scratching) reflects increased cytokines in the psoriatic lesions and perilesional skin. Subsequently, an inflammatory response directed against melanocyte occurs in the draining lymph node, and specific $\mathrm{T}$ cells (cytotoxic) migrate by adhering to their vascular adhesion receptors towards the area (T8-T12 dermatome in our patient) where vulnerable melanocytes (due to somatic mosaicism) are residing. ${ }^{14}$ This destruction of melanocytes caused the clinically observed cutaneous depigmentation.

\section{Treatment}

Currently, there is still no standard therapy due to the paucity of cases. However, the management of these two diseases is similar. A case report of non- segmental vitiligo preceding psoriasis with exclusive colocalization, documented improvement of psoriasis but not of vitiligo with the combination of narrowband UVB and a topical steroid. ${ }^{16}$

\section{CONCLUSION AND RECOMMENDATION}

The commonly reported type of vitiligo concurrent to psoriasis is the non-segmental type. Several theories were suggested to explain the coexistence of the two conditions such as altered cytokine milieu, Koebner phenomenon, and autoimmunity. To date, the exact pathologic relationship has not yet been fully elucidated. Hence, further research is necessary to define the etiologic link leading to the concurrence and colocalization of these common dermatoses.

This is a case of rare presentation of coexisting and colocalized psoriasis and segmental type vitiligo. A recent study suggested an overlapping mechanism of immunemediated destruction of vulnerable melanocytes for segmental type vitiligo. In our case, the altered local cytokine milieu resulting from the psoriatic flare may have initiated the inflammatory reaction against truncal melanocytes with 
acquired somatic mosaicism. This is very likely since psoriasis preceded the appearance of the solitary depigmented patch during adulthood in contrast to most cases of segmental vitiligo with documented onset during early years of life. Because an autoimmune etiology cannot be fully discounted, it is still important to rule out presence of other concomitant autoimmune diseases through a thorough history, complete physical examination and appropriate ancillary tests.

The combination of topical steroids and narrowband UVB is effective in the treatment of psoriasis. In contrast, segmental vitiligo responds poorly to topical therapy using steroid and tacrolimus with phototherapy. Lastly, precaution must be observed upon use of phototherapy as a treatment for both psoriasis and vitiligo where the substantial risk of burn can occur if colocalized depigmented lesions are not reassessed completely.

\section{Statement of Authorship}

All authors approved the final version submitted.

\section{Author Disclosure}

All authors declared no conflicts of interest.

\section{Funding Source}

This paper was funded by the authors. No external funding agency.

\section{REFERENCES}

1. Bakar-Dertlioğlu S, Ucak H, Cicek D, Bitiren M. Coexistence of vitiligo and psoriasis: Three case reports. Turk J Pediatr. 2012;54:77-9.

2. Koransky JS, Roenigk HH. Vitiligo and psoriasis.J Am Acad Dermatol. 1982;7(2):183-9.
3. Papadavid E, Yu RC, Munn S, Chu AC. Strict anatomical coexistence of vitiligo and psoriasis vulgaris - a Koebner phenomenon? Clin Exp Dermatol. 1996;21(2):138-40.

4. Van de Kerkhof P, Nestle F. Psoriasis. In: : Bolognia JL, Jorizzo JL, \& Schaffer JV, eds Dermatology. 3rd ed. Philadelphia: Elsevier Saunders; 2012. p. 135-55.

5. Inadamar A, Sampagi V, Athanikar S, Patil M, Deshmukh N. Vitiligo and psoriasis: Coexistence with colocalization. Indian J Dermatol Venereol. 2001;(67):214-5.

6. Berger T, Kiesewetter F, Maczek C, Bauer N, Lueftl M, Schuler $\mathrm{G}$, et al. Psoriasis confined strictly to vitiligo areas - a Koebner-like phenomenon? J Eur Acad Dermatol Venereol. 2006;20(2):178-83.

7. Percivalle S, Piccinno R, Caccialanza M. Concurence of vitiligo and psoriasis: a simple coincidence? Clin Exp Dermatol. 2009;34:90-1.

8. Campanati A, Giuliodori K, Ganzetti G, Liberati G, Offidani AM. A Patient with Psoriasis and Vitiligo Treated with Etanercept: Am J Clin Dermatol. 2010;11:46-8.

9. Bassiouny DA, Shaker O. Role of interleukin-17 in the pathogenesis of vitiligo: Role of IL-17 in the pathogenesis of vitiligo. Clin Exp Dermatol. 2011;36(3):292-7.

10. Dhar S, Malakar S, Dhar S. Colocalization of vitiligo and psoriasis in a 9-year-old boy. Pediatr Dermatol. 2009;15(3):242-3.

11. Chakraborty D, Haneef N, Razvi F, Kumar B, Fatima N, Koganti N, et al. A case report showing coexistence of two autoimmune diseasespsoriasis and vitiligo. J Med Allied Sci. 2017;7(2):114-7.

12. Langley AR, Manley P, Asai Y. A Case of Colocalized Vitiligo and Psoriasis. J Cutan Med Surg. 2016;2:150-2.

13. Castro CCS de. Prevalence of psoriasis in a study of 261 patients with vitiligo. An Bras Dermatol. 2005;80(5):489-92.

14. van Geel N, Speeckaert R. Segmental Vitiligo. Dermatol Clin. 2017;35(2):145-50.

15. Gilbert S. The Neural Crest [Internet]. 6th ed. Massachusetts: Sinauer Associates, Inc., Publishers; 2000 [cited 2018 Jul 22]. Available from: http://www.ncbi.nlm.nih.gov/books/NBK10065

16. Moreno MI, Moreno LH. Coexistence of psoriasis and vitiligo. Colomb Med. 2009;39(4):378-83.

\title{
Have you read the current trends in
} Medical and Health Research in the Philippines?

\section{Acta Medica Philippina The National Health Science Journal}

\author{
Access Online: www.actamedicaphilippina.org
}

